\title{
Genetic diversity of Saccharum spontaneum from geographical regions of China assessed by simple sequence repeats
}

L.N. Fan, H.H. Deng, Q.W. Luo, H.Y. He, Y. Li, Q.N. J.T. Wu, Q.W. Li, S.M. Liu and Y.W. Qi

Guangdong Key Laboratory of Sugarcane Improvement and Biorefinery, Guangzhou Sugarcane Industry Research Institute, Guangzhou, China

Corresponding author: Y.W. Qi

E-mail: yongwen2001@163.com

Genet. Mol. Res. 12 (4): 5916-5925 (2013)

Received August 8, 2012

Accepted September 5, 2013

Published November 26, 2013

DOI http://dx.doi.org/10.4238/2013.November.26.1

\begin{abstract}
Saccharum spontaneum is the most variable wild relative of sugarcane with potential for use in sugarcane improvement programs. In order to help preserve and exploit this species, 152 accessions from eight major geographical regions in China, including Hainan, Guangdong, Guangxi, Yunnan, Sichuan, Guizhou, Fujian, and Jiangxi provinces, were investigated by analyzing 20 simple sequence repeats (SSRs), including 11 genomic SSRs (gSSRs) and nine SSRs developed from expressed sequence tags (EST-SSRs). A total of 454 alleles were generated by the 20 SSRs, with 295 and 159 alleles detected by gSSRs and EST-SSRs respectively. The Mantel test showed significant correlation between genetic matrixes among the studied accessions revealed by gSSRs versus EST-SSRs, although the average polymorphism of EST-SSRs (17.7) was much lower than that of gSSRs (26.8). Among the eight provinces, collections from Guizhou were the most diverse and those from Guangdong were the most distinct. Clustering analysis and principal component analysis accordantly classified the accessions into four groups, which were "Southwest
\end{abstract}


group", "Hainan group", "Guangdong group", and "Guangxi group", based on the geographical origin of the major accessions in each group, demonstrating that geographical factors play an important role in the pattern of genetic structure of Chinese S. spontaneum. As two (Guizhou and Yunnan) of the three provinces with highest genetic diversity are located in southwest China, we concluded that southwest China is the region with the highest genetic diversity of $S$. spontaneum.

Key words: Saccharum spontaneum L.; China; Genetic diversity; SSR; EST-SSR

\section{INTRODUCTION}

Saccharum spontaneum L. is a wild relative of sugarcane cultivar (Saccharum spp) with high levels of disease resistance, adaptability and stress tolerance (Bremer, 1961), which has been successfully exploited in sugarcane improvement programs since 1915 by hybrid breeding with Saccharum officinarum. It has been estimated that an average of $15-25 \%$ of chromatin in commercial sugarcane is derived from S. spontaneum (Sreenivasan et al., 1987; Glaszmann et al., 1989; D'Hont et al., 1993). In view of its importance in sugarcane improvement programs, there have been many concerted efforts to collect and assess $S$. spontaneum germplasm, and to exploit its genetic potential in sugarcane breeding (Tai and Miller, 2001; Amalraj et al., 2006; Mary et al., 2006; Qi et al., 2009).

In China, S. spontaneum is widespread within the geographical range of northern latitudes of $18^{\circ}-33^{\circ}$ and eastern longitudes of $97^{\circ}-122^{\circ}$ (Chen and Phillips, 2006). Native $S$. spontaneum has been used in sugarcane improvement in China since the 1950s (Deng and Zhang, 2006). Through interspecific hybrid breeding of $S$. spontaneum with $S$. officinarum and the offspring's backcrossing with Saccharum spp, many elite "Yacheng" lines with high yield, high sucrose, or resistance to some disease or abiotic stress have been developed, some of which have been the major parental resources in mainland China's sugarcane breeding program (Lao et al., 2008; Zhang et al., 2009). For better preservation and utilization of S. spontaneum, several studies on the genetic diversity of Chinese $S$. spontaneum have been reported. An early study indicated that most clones of Chinese $S$. spontaneum from the southwest region were $2 \mathrm{n}=$ 80 , and some $2 n=64$, in addition to rare ones being $2 n=68$ or 116 (Chen et al., 1981). In recent years, using DNA markers such as RAPD and SRAP, the genetic diversity of $S$. spontaneum in some geographical regions in China was also investigated (Zhang et al., 2010; Chang et al., 2012), which showed that there was substantial genetic variation within the accessions studied.

Simple sequence repeats (SSR) or microsatellites are short fragments of DNA that consist of small motifs of one to six tandem repeat base pairs (Wang et al., 1994; Jarne and Lagoda, 1996). EST-SSR is an SSR developed through SSR mining in expressed sequence tags (EST) obtained from partial sequencing of cDNAs (Scott et al., 2000; Thiel et al., 2003). As ESTs represent part of expressed genes, they can provide direct mapping of genes with known functions, allowing candidate genes involved in important metabolic pathways to be directly evaluated for associations with important agronomic traits (Liang et al., 2000; Cato et al., 2001; Ellis and Burke, 2007). EST-SSRs have been widely used for germplasm analysis, cultivar identification, parent evaluation, genetic mapping, and marker-assisted selection in 
many crops including Saccharum species (Cordeiro et al., 2001; Yu et al., 2004; Han et al., 2006; Sraphet et al., 2011).

In the present study, $152 \mathrm{~S}$. spontaneum accessions from eight geographical regions, namely Hainan, Guangdong, Guangxi, Fujian, Yunnan, Sichuan, Guizhou and Jiangxi Provinces, were genotyped by SSRs. These eight provinces, located in the south and southwest China, are the major distributed regions of $S$. spontaneum in China. The objectives of this study were to compare the allelic variations and discrimination power of the two types of SSR in S. spontaneum, to reveal the genetic diversity and structure of Chinese S. spontaneum, and then to provide information for germplasm collection and use in the future.

\section{MATERIAL AND METHODS}

\section{Plant materials}

A total of 152 S. spontaneum accessions were selected from eight geographical regions: Hainan, Guangdong, Guangxi, Yunnan, Sichuan, Guizhou, Fujian, and Jiangxi Provinces (Figure 1). These accessions were maintained in the Guangdong Sugarcane Germplasm Bank, Sanya, Hainan Province, China.

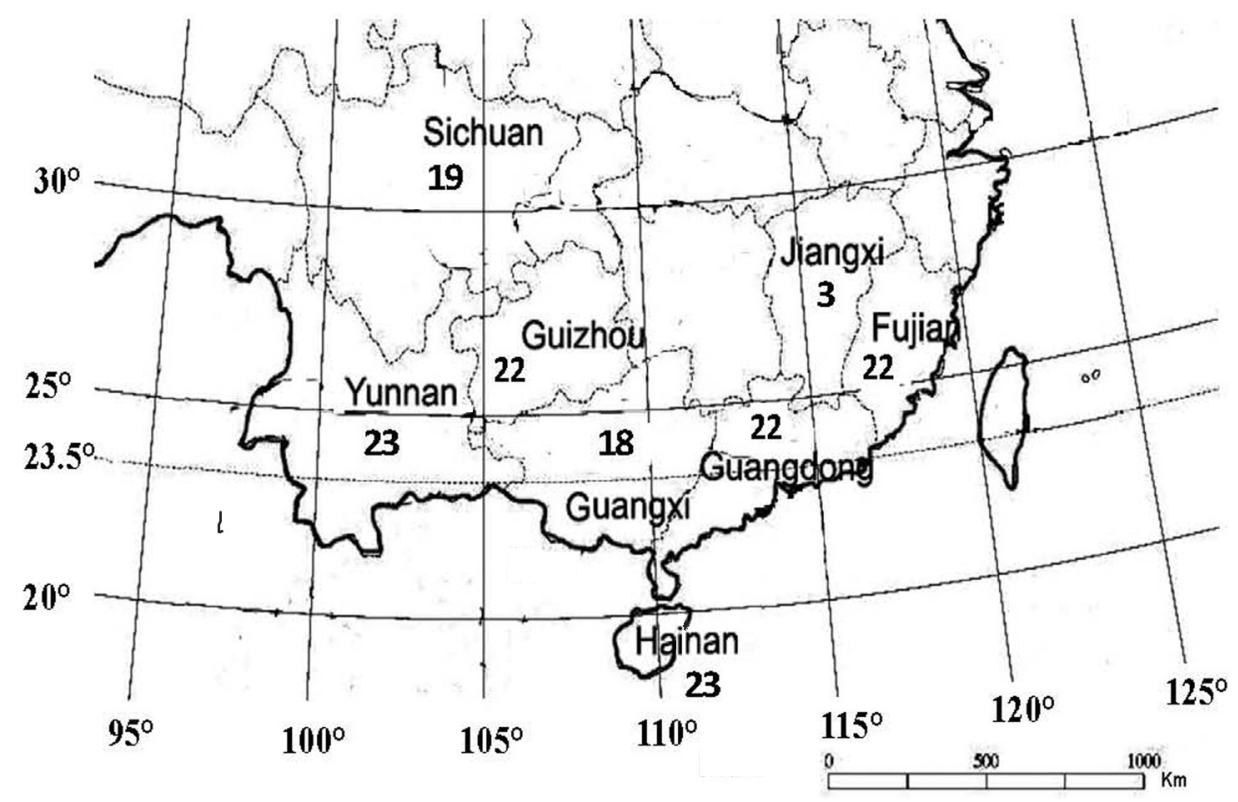

Figure 1. Map of the provinces in China and the number of Saccharum spontaneum clones studied in each province.

\section{DNA extraction and microsatellite genotyping}

DNA was extracted from young leaf tissue using a modified CTAB procedure (Hoisington, 1992). DNA concentration was estimated by $0.75 \%$ agarose gel electrophoresis along with standard DNA, followed by dilution before PCR amplification. 
A total of 20 SSR including $11 \mathrm{gSSRs}$ from the International Consortium of Sugarcane Biotechnology (Cordeiro et al., 2001; Pan, 2006) and 9 EST-SSRs reported by Pinto et al. (2004) were used in this study (Table 1). The PCR volume was $25 \mu \mathrm{L}$ containing $30 \mathrm{ng}$ DNA template, 1.0 U Taq DNA polymerase, $2.5 \mu \mathrm{L}$ 10X PCR buffer, $2.5 \mu \mathrm{L} 25 \mathrm{mM} \mathrm{Mg}^{2+}, 0.25 \mathrm{mM}$ each dNTP, and $0.3 \mu \mathrm{M}$ each forward and reverse primer. Amplifications were carried out in a Peltier Thermal Cycler PTC-200 (Bio-Rad, Watertown, MA, USA) with the following profile: initial denaturation at $94^{\circ} \mathrm{C}$ for $4 \mathrm{~min}$, followed by 30 cycles of $94^{\circ} \mathrm{C}$ for $30 \mathrm{~s}, 54^{\circ} \mathrm{C}$ for $30 \mathrm{~s}$, and $72^{\circ} \mathrm{C}$ for $60 \mathrm{~s}$, and a final extension at $72^{\circ} \mathrm{C}$ for $5 \mathrm{~min}$. Amplified DNA fragments were resolved by electrophoreses on $8 \%$ denaturing polyacrylamide gels at $70 \mathrm{~W}$ for $2 \mathrm{~h}$ and revealed with silver staining (Bassam et al., 1991).

\begin{tabular}{|c|c|c|c|c|c|}
\hline Name & SSR type & Total bands & Polymorphic bands & Genetic diversity index & PIC \\
\hline mSSCIR58 & gSSR & 31 & 30 & 0.2100 & 0.1817 \\
\hline $\mathrm{SMC} 21 \mathrm{Sa}$ & gSSR & 20 & 12 & 0.2381 & 0.2014 \\
\hline SMC597CS & gSSR & 29 & 27 & 0.2645 & 0.2221 \\
\hline SMC668CS & gSSR & 25 & 18 & 0.2834 & 0.2344 \\
\hline SMC720BS & gSSR & 27 & 22 & 0.2610 & 0.2181 \\
\hline SMC851MS & gSSR & 43 & 31 & 0.2028 & 0.1756 \\
\hline SSCIR15 & gSSR & 33 & 25 & 0.2170 & 0.1853 \\
\hline SSCIR16 & gSSR & 15 & 8 & 0.2081 & 0.1792 \\
\hline SSCIR36 & gSSR & 39 & 35 & 0.2473 & 0.2093 \\
\hline SSCIR43 & gSSR & 16 & 14 & 0.2691 & 0.2189 \\
\hline SMC18251a & gSSR & 17 & 17 & 0.2291 & 0.1945 \\
\hline SCA03 & gSSR & 36 & 16 & 0.3424 & 0.2730 \\
\hline SCA04 & EST-SSR & 14 & 14 & 0.2624 & 0.2216 \\
\hline SCA07 & EST-SSR & 24 & 19 & 0.2542 & 0.2112 \\
\hline SCB01 & EST-SSR & 15 & 14 & 0.2194 & 0.1842 \\
\hline SCB02 & EST-SSR & 4 & 4 & 0.2138 & 0.1860 \\
\hline $\mathrm{SCC} 02$ & EST-SSR & 14 & 9 & 0.2736 & 0.2287 \\
\hline $\mathrm{SCC} 05$ & EST-SSR & 30 & 23 & 0.3055 & 0.2475 \\
\hline $\mathrm{SCC} 07$ & EST-SSR & 10 & 8 & 0.3323 & 0.2671 \\
\hline SCC10 & EST-SSR & 12 & 9 & 0.2344 & 0.1989 \\
\hline
\end{tabular}

\section{Data analysis}

The sugarcane clones were scored for the presence (1) or absence (0) of common bands in the different patterns. The amplified fragments produced by the SSRs were considered alleles of a single locus. Genetic distance (GS) were calculated for all possible pairwise comparisons using the Jaccard method (1908): $\mathrm{Jij}=1-\mathrm{A} /(\mathrm{A}+\mathrm{B}+\mathrm{C})$, where A was the number of bands common to genotype $\mathrm{B}$ and $\mathrm{C}, \mathrm{B}$ was the number of bands unique to the first genotype, and $\mathrm{C}$ was the number of bands unique to the second genotype, using the software package NTSys-pc (Rohlf, 1995). A phylogenetic tree was constructed on the basis of the neighbor-joining method implemented in PowerMarker version 2.7 (Liu and Muse, 2005), and the unrooted phylogenetic tree was finally schematized with the software MEGA ver. 3.1 (Tamura et al., 2007). A principal component analysis (PCA) was performed on the basis of similarity measures to cluster these 96 accessions into major groups using NTSys-pc. Genetic differentiation (Gst) at SSRs loci between the provinces was estimates by F-statistics (Nei, 1973) in Popgene software ver. 1.31 (Yeh et al., 1999). The Mantel test was conducted to assess the correlation between the genetic distance matrices between the accessions revealed by gSSRs and EST-SSRs in PowerMarker. 


\section{RESULTS}

\section{Allelic diversity of the gSSRs and EST-SSRs}

A total of 295 alleles were detected by 11 gSSRs, of which 239 were polymorphic (Table 1). Allele number ranged from 15 (SSCIR16) to 43 (SMC851MS) with a mean of 26.82 alleles per gSSR. For the $11 \mathrm{gSSRs}$, genetic diversity index varied from 0.2028 to 0.2834 with an average of 0.2391 , and the polymorphic information content (PIC) ranged from 0.1756 to 0.2344 with an average of 0.2018 .

The 9 EST-SSRs detected 159 alleles among the clones studied, of which 116 (73.0\%) were polymorphic. The number of alleles varied from 4 (SCB02) to 36 (SCA03), with a mean of 17.67 alleles per EST-SSR. Genetic diversity index of the EST-SSRs varied from 0.2138 to 0.3424 with an average of 0.2708 , and the PIC ranged from 0.1842 to 0.2730 with an average of 0.2242 .

Overall, the polymorphism obtained with gSSRs was much higher than that obtained with EST-SSRs. Of the 5 SSRs with polymorphism less than 10, 4 (80\%) were EST-SSRs and only $1(20 \%)$ was a gSSR. The average number of alleles detected by EST-SSRs (12.89) was about $59.30 \%$ of that by gSSRs (21.72). In contrast to the lower polymorphism, EST-SSRs showed a higher genetic diversity value than gSSRs. The average genetic diversity index of EST-SSRs (0.2708) was $17.02 \%$ higher than that of gSSRs (0.2391).

\section{Genetic diversity of $S$. spontaneum in the eight geographical regions}

Table 2 shows that 126-316 alleles were detected in the eight regions. Because only three accessions from Jiangxi were genotyped, the number of alleles detected in Jiangxi was the least. Among the other seven provinces, the highest number of alleles was detected in Guizhou, followed by Fujian and Yunnan, whereas Hainan, Guangdong, and Guangxi accessions showed less polymorphism. Comparison of the allelic distribution across the eight provinces indicated that only 3 special alleles could be detected in only one province, so the origin of $S$. spontaneum accessions could not be discriminated using specific alleles. Of the eight provinces studied, genetic diversity index of accessions from Guangdong, Yunnan, and Sichuan was the highest, and that of Jiangxi, Guangxi, and Hainan was the lowest (Table 2).

\begin{tabular}{|c|c|c|c|}
\hline Province & No. of accessions & Polymorphic alleles & Genetic diversity index \\
\hline Fujian & 22 & 312 & 0.2375 \\
\hline Guangdong & 22 & 297 & 0.2459 \\
\hline Guangxi & 18 & 261 & 0.1794 \\
\hline Guizhou & 22 & 316 & 0.2421 \\
\hline Hainan & 23 & 284 & 0.2343 \\
\hline Jiangxi & 3 & 126 & 0.1540 \\
\hline Sichuan & 19 & 303 & 0.2421 \\
\hline Yunnan & 23 & 311 & 0.2429 \\
\hline Total & 152 & 355 & 0.2223 \\
\hline
\end{tabular}


Pairwise genetic distances between the eight provinces were calculated (Table 3 ). The maximum distance $(0.2690)$ was found between Guangdong and Yunnan and the smallest distance (0.1975) was observed between Jiangxi and Guangxi. Overall, Guangdong showed the largest genetic difference to the other provinces, with an average Jaccard distance of 0.2582 , followed by Yunnan (0.2574), and Fujian (0.2566), whereas, Jiangxi (0.2389), Guangxi (0.2449), and Hainan (0.2550) displayed the smallest genetic distance to the other provinces.

Table 3. Pair-wise genetic distances among the accessions of the eight provinces.

\begin{tabular}{llllllllc}
\hline & Fujian & Guangdong & Guangxi & Guizhou & Hainan & Jiangxi & Sichuan & Yunnan \\
\hline Fujian & 0 & 0.264 & 0.2629 & 0.2561 & 0.2594 & 0.2428 & 0.2534 & 0.2573 \\
Guangdong & 0.264 & 0 & 0.2459 & 0.2638 & 0.2452 & 0.2464 & 0.2643 & 0.2690 \\
Guangxi & 0.2629 & 0.2459 & 0 & 0.2537 & 0.2491 & 0.1975 & 0.2516 & 0.2537 \\
Guizhou & 0.2561 & 0.2638 & 0.2537 & 0 & 0.2586 & 0.2458 & 0.2554 & 0.2576 \\
Hainan & 0.2594 & 0.2452 & 0.2491 & 0.2586 & 0 & 0.2458 & 0.2578 & 0.2599 \\
Jiangxi & 0.2428 & 0.2464 & 0.1975 & 0.2458 & 0.2458 & 0 & 0.2476 & 0.2465 \\
Sichuan & 0.2534 & 0.2643 & 0.2516 & 0.2554 & 0.2578 & 0.2476 & 0 & 0.2581 \\
Yunnan & 0.2573 & 0.2690 & 0.2537 & 0.2576 & 0.2599 & 0.2465 & 0.2581 & 0 \\
\hline
\end{tabular}

For the 355 polymorphic alleles, Gst of 321 alleles (90.4\%) across the eight provinces was lower than 0.2 , and that of 34 alleles $(9.6 \%)$ was higher than 0.2 . The overall average GST of the polymorphic alleles was 0.1146 . gSSRs showed a higher genetic differentiation (0.1197) than did EST-SSRs (0.1095).

\section{Genetic structure within Chinese $S$. spontaneum}

Clustering analysis based on neighbor-joining method indicated that the 153 clones were classified into four genetically distinct groups (Figure 2). Group-i was represented by 48 accessions, of which 19, 6 and 10 accessions were from Yunnan, Sichuan, and Guizhou, respectively. Accessions from the three southwest provinces accounted for about $73 \%$ of the total Group-i accessions. Therefore, Group-i was characteristic of the "Southwest group". Group-ii with 30 accessions was populated by accessions collected predominantly from Hainan Province. Of the 23 Hainan accessions studied, 20 were classified into Group-ii, accounting for about $66.7 \%$ of the total Group-accessions, so Group-ii was characteristic of the "Hainan group". Group-iii consisted of 24 accessions, of which 12 were Guangdong accessions accounting for $50 \%$ of this group's accessions, so Group-iii was characteristic of the "Guangdong group". The most obvious feature of the Group-iv was that nearly all Guangxi accessions were classified into Group-iv, accounting about $63 \%$ of the total accessions of the group, so Group-iv was identified as the "Guangxi group". The 22 Fujian accessions were dispersed in all four groups, and the 3 Jiangxi accessions were classified into the "Guangdong group" and "Guangxi group". No special group was found for the accessions from these two provinces.

In PCA analysis, the first three PCA axes accounted for 5.5, 3.8, and 3.2\% of the total variances, respectively. PCA analysis also classified most of the accessions into four groups in agreement with that by clustering analysis (Figure 3). The concordant results by clustering analysis and PCA analysis suggested that there was an obvious genetic structure related to geographical regions within Chinese $S$. spontaneum. 


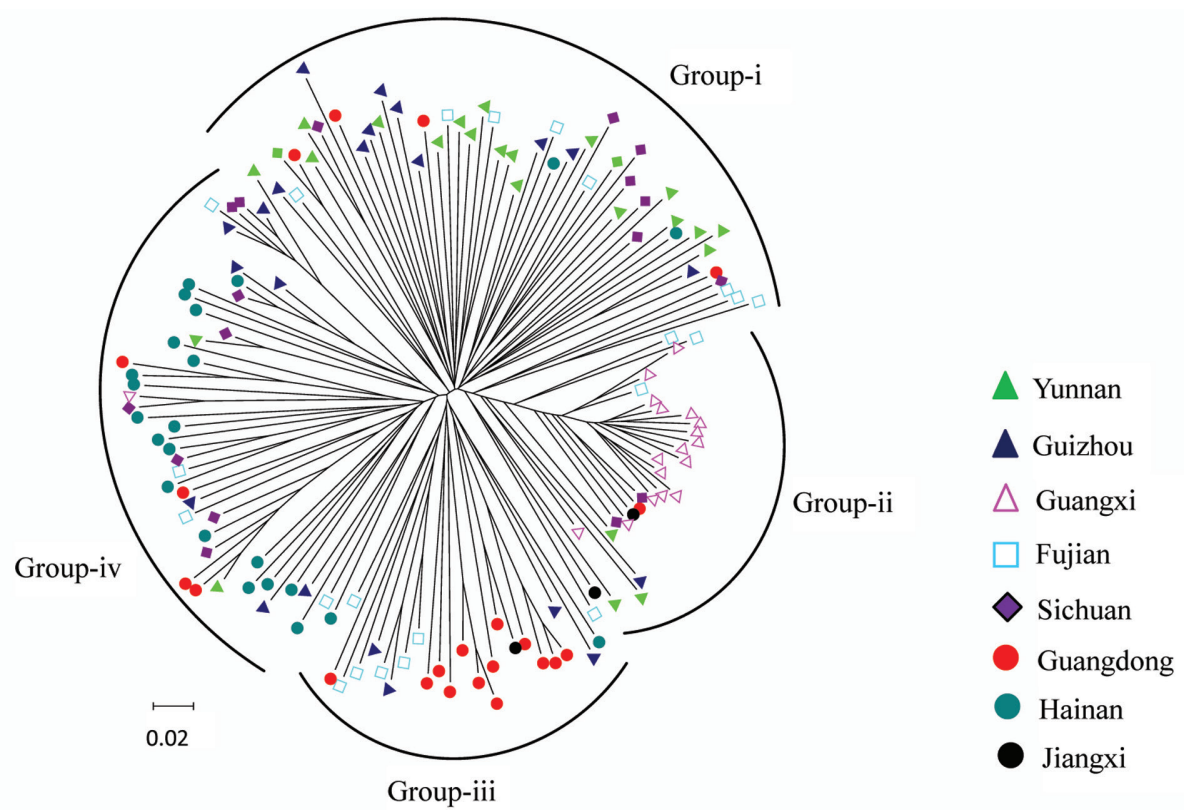

Figure 2. Unrooted phylogram based on neighbor-joining tree by Jaccard's genetic distance among the accessions from eight provinces.

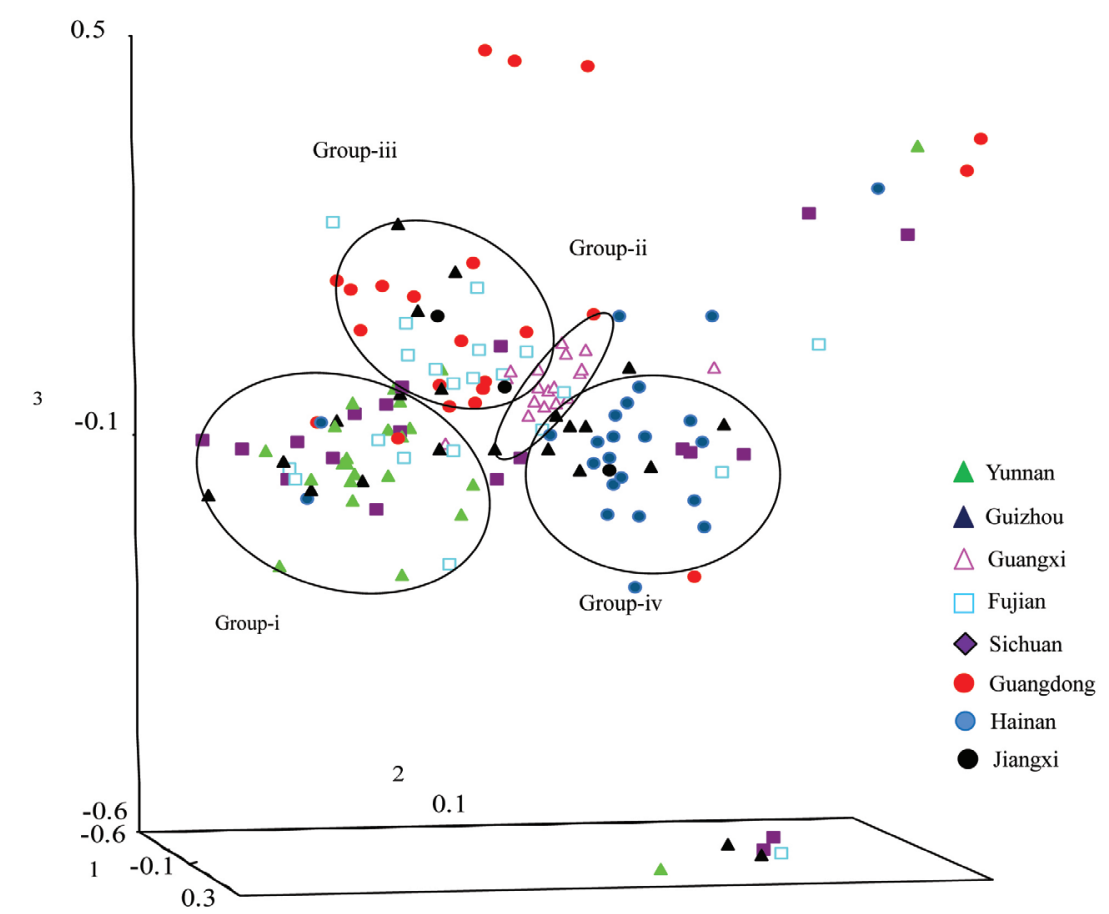

Figure 3. Principle component analysis of the accessions from eight provinces. 


\section{DISCUSSION}

The development of EST-SSR markers was particularly attractive because they represent coding regions of the genome, which allows for a direct association between genes and important agronomic traits (Cato et al., 2001; Thiel et al., 2003). In sugarcane, many EST-SSRs have been developed and used in genetic analysis (Pinto et al., 2004; Pinto et al., 2006; Marconi et al., 2011). However, the previous studies about EST-SSRs in sugarcane were mainly conducted on Saccharum spp, although several wild species including S. spontaneum were included. This study comparatively analyzed the allelic diversity of EST-SSRs and gSSRs within many more $S$. spontaneum accessions than in other reported studies. In S. spontaneum, polymorphism of EST-SSRs was also much lower than that of gSSRs, which was in agreement with the previous studies in Saccharum and other crops. Of the 9 EST-SSRs used in this study, only 1 EST-SSR (11.1\%) was found with polymorphism higher than 20, whereas there were 6 gSSRs $(54.5 \%)$ of the total $11 \mathrm{gSSRs}$ with polymorphism of more than 20 . The average polymorphism of EST-SSRs to that of gSSRs $(59.3 \%)$ was lower than that $(75.8 \%)$ in other Saccharum species (Pinto et al., 2006). However, although the polymorphism of the two types of SSRs was much different, this study found that there was significant correlation between genetic relationship matrices between the $S$. spontaneum accessions revealed by gSSRs and EST-SSRs, respectively (Mantel test, $\mathrm{r}=0.7953, \mathrm{P}<0.05$ ), suggesting little difference when assessing genetic relationships between Saccharum clones by these two types of SSRs, which was also in agreement with the previous work of Pinto et al. (2006) and Marconi et al. (2011).

$S$. spontaneum has been successfully used in China sugarcane breeding programs for many years (Deng and Zhang, 2006; Zhang et al., 2009), but less is known about the genetic diversity of Chinese $S$. spontaneum, especially the genetic difference in the collections across geographical regions. Therefore, the genetic diversity of the $S$. spontaneum accessions from eight major distributed regions in China was investigated in this study. In genotyping the accessions by both gSSRs and EST-SSRs, this study showed that Guizhou, Fujian, and Yunnan were the provinces with the largest genetic diversity and that Jiangxi, Guangxi, and Hainan were the provinces with the lowest genetic diversity. Since two of the three provinces with the largest genetic diversity (Guizhou and Yunnan) are located in southwest China, this study suggests that this is the region with the largest genetic diversity of S. spontaneum in China.

Hainan Province differs from other provinces because it is separated from other provinces by a strait (Figure 1). Hainan is at least $190 \mathrm{~km}$ from other provinces, so it is difficult for pollen or stems to be transported between Hainan S. spontaneum and other S. spontaneum. This may be the reason for the low diversity of Hainan accessions found in this study. In addition to lower diversity, no special alleles found in Hainan accessions indicated that Hainan S. spontaneum was introduced from other regions. This study found that Guangdong S. spontaneum showed the closest genetic relationship to Hainan accessions (Table 3). Indeed, Guangdong is the nearest province to Hainan (Figure 1). The concordant results suggest that Hainan S. spontaneum originally came from Guangdong.

This study showed that there was obvious genetic structure related to geographical origin within China's S. spontaneum. The classification analysis and PCA analysis concordantly identified four groups as the "Southwest group", "Guangdong group", "Guangxi group", and "Hainan group" in the accessions studied. Similar results were also found in other studies. In previous studies on Chinese S. spontaneum (Chang et al., 2012) and Indian S. spontaneum 
(Mary et al., 2006), it was also found that the genetic relationship between the accessions of $S$. spontaneum was related to geographical distribution and ecological conditions. All of these studies indicated that geographical factors play an important role in the pattern of the genetic structure within S. spontaneum. Therefore, when collecting S. spontaneum, more attention should be paid to the accessions with agronomic traits or growth habits related to local ecological conditions to enhance the genetic diversity of conserved accessions.

\section{ACKNOWLEDGMENTS}

Research supported by the National Natural Science Foundation of China (\#30800700), the Earmarked Fund for Modern Agro-industry Technology Research System (\#CARS-20-14), and the Guangdong Agricultural Science and Technology Plan (\#2011B060400019).

\section{REFERENCES}

Amalraj VA, Balakrishnan R, Jebadhas AW and Balasundaram N (2006). Constituting a core collection of Saccharum spontaneum L. and comparison of three stratified random sampling procedures. Genet. Resour. Crop Evol. 53: 15631572.

Bassam BJ, Caetano-Anolles G and Gresshoff PM (1991). Fast and sensitive silver staining of DNA in polyacrylamide gels. Anal. Biochem. 196: 80-83.

Bremer G (1961). Problems in breeding and cytology of sugar cane. Euphytica 10: 59-78.

Cato SA, Gardner RC, Kent J and Richardson TE (2001). A rapid PCR-based method for genetically mapping ESTs. Theor. Appl. Genet. 102: 296-306.

Chang D, Yang FY, Yan JJ, Wu YQ, et al. (2012). SRAP analysis of genetic diversity of nine native populations of wild sugarcane, Saccharum spontaneum from Sichuan, China. Genet. Mol. Res. 11: 1245-1253.

Chen CL, Li LF and Wu SZ (1981). Chromosome number distribution of Saccharum spontaneum in the southwest region of China. Intl. Sugar J. 83: 264-267.

Chen SL and Phillips SM (2006). Saccharum Linnaeus. In: Flora of China Vol. 22 (Poaceae) (Wu ZY, Raven PH and Hong DY, eds.). Missouri Botanical Garden Press, St. Louis., 576-581.

Cordeiro GM, Casu R, McIntyre CL, Manners JM, et al. (2001). Microsatellite markers from sugarcane (Saccharum spp.) ESTs cross transferable to Erianthus and Sorghum. Plant Sci. 160: 1115-1123.

D’Hont A, Lu YH, Feldmann P and Glaszmann JC (1993). Cytoplasmic diversity in sugarcane revealed by heterologous probes. Sugarcane 1: 12-15.

Deng HH and Zhang Q (2006). Analysis on the parents of commercial varieties released in Mainland China in recent years. Guangdong Agric. Sci. 12: 7-10.

Ellis JR and Burke JM (2007). EST-SSRs as a resource for population genetic analyses. Heredity 99: 125-132.

Glaszmann JC, Fautret A, Noyer JL, Feldmann P, et al. (1989). Biochemical genetic markers in sugarcane. Theor. Appl. Genet. 78: 537-543.

Han Z, Wang C, Song X, Guo W, et al. (2006). Characteristics, development and mapping of Gossypium hirsutum derived EST-SSRs in allotetraploid cotton. Theor. Appl. Genet. 112: 430-439.

Hoisington D (1992). Laboratory Protocols. CIMMYT Applied Molecular Genetics Laboratory. Mexico, D.F. CIMMYT. Jaccard P (1908). Nouvelles recherches sur la distribution florale. Bull. Soc. Vaudoise Sci. Nat. 44: 223-270.

Jarne P and Lagoda PJL (1996). Microsatellites, from molecules to populations and back. Trends Ecol. Evol. 11: 424-429.

Lao FY, Liu R, He HY, Deng HH, et al. (2008). AFLP analysis of genetic diversity in series sugarcane parents developed at HSBS. Fenzi Zhiwu Yuzhong 6: 517-522.

Liang F, Holt I, Pertea G, Karamycheva S, et al. (2000). An optimized protocol for analysis of EST sequences. Nucleic Acids Res. 28: 3657-3665.

Liu K and Muse SV (2005). PowerMarker: an integrated analysis environment for genetic marker analysis. Bioinformatics 21: $2128-2129$

Marconi TG, Costa EA, Miranda HR, Mancini MC, et al. (2011). Functional markers for gene mapping and genetic diversity studies in sugarcane. BMC Res. Notes 4: 264.

Mary S, Nair N, Chaturvedi PK and Selvi A (2006). Analysis of genetic diversity among Saccharum spontaneum L. from 
four geographical regions of India, using molecular markers. Genet. Resour. Crop Evol. 53: 1221-1231.

Nei M (1973). Analysis of gene diversity in subdivided populations. Proc. Natl. Acad. Sci. U. S. A. 70: 3321-3323.

Pan YB (2006). Highly polymorphic microsatellite DNA markers for sugarcane germplasm evaluation and variety identity testing. Sugar Tech 8: 246-256.

Pinto LR, Oliveira KM, Marconi T, Garcia AAF, et al. (2006). Characterization of novel sugarcane expressed sequence tag microsatellites and their comparison with genomic SSRs. Plant Breed. 125: 378-384.

Pinto LR, Oliveira KM, Ulian EC, Garcia AA, et al. (2004). Survey in the sugarcane expressed sequence tag database (SUCEST) for simple sequence repeats. Genome 47: 795-804.

Qi Y, Fan LN, He HY, Chen YS, et al. (2009). Genetic diversity assessment of Saccharum spontaneum L. native to Guangdong area with agronomic traits. Sugarcane and Canesugar 3: 7-10.

Rohlf FJ (1995). NTSYS-pc Numerical Taxonomy and Multivariate Analysis System, Version 2.1. User Guide. Exeter Software, Setauket.

Scott KD, Eggler P, Seaton G, Rossetto M, et al. (2000). Analysis of SSRs derived from grape ESTs. Theor. Appl. Genet. 100: 723-726.

Sraphet S, Boonchanawiwat A, Thanyasiriwat T, Boonseng O, et al. (2011). SSR and EST-SSR-based genetic linkage map of cassava (Manihot esculenta Crantz). Theor. Appl. Genet. 122: 1161-1170.

Sreenivasan TV, Ahloowalia BS and Heinz DJ (1987). Cytogenetics. In: Sugarcane Improvement Through Breeding (Heinz D, ed.). Elsevier, Amsterdan, 211-253.

Tai PYP and Miller JD (2001). A core collection for Saccharum spontaneum L. from the world collection of sugarcane. Crop Sci. 41: 879-885.

Tamura K, Dudley J, Nei M and Kumar S (2007). MEGA4: Molecular Evolutionary Genetics Analysis (MEGA) software version 4.0. Mol. Biol. Evol. 24: 1596-1599.

Thiel T, Michalek W, Varshney RK and Graner A (2003). Exploiting EST databases for the development and characterization of gene-derived SSR-markers in barley (Hordeum vulgare L.). Theor. Appl. Genet. 106: 411-422.

Wang Z, Weber JL, Zhong G and Tanksley SD (1994). Survey of plant short tandem DNA repeats. Theor. Appl. Genet. 88: 1-6.

Yeh FC, Yang RC and Boyle T (1999). POPGENE, Version 1.31. Microsoft Window-based Freeware for Population Genetic Analysis. Quick User Guide. University of Alberta, Alberta, Canada.

Yu JK, La RM, Kantety RV and Sorrells ME (2004). EST derived SSR markers for comparative mapping in wheat and rice. Mol. Genet. Genomics 271: 742-751.

Zhang GM, Li YR, He WZ, He H, et al. (2010). Analysis of the genetic diversity in Saccharum spontaneum L. accessions from Guangxi Province of China with RAPD-PCR. Sugar Tech 12: 31-35.

Zhang Q, Qi YW, Zhang CM, Chen YS, et al. (2009). Pedigree analysis of genetic relationship among core parents of sugarcane in Mainland China. Guangdong Agric. Sci. 10: 44-48. 